\title{
P101 'A FO0T IN THE DOOR' - AN INTRODUCTORY PROGRAMIME FOR PATIENTS WITH EARLY STAGE MOTOR NEURON DISEASE
}

Nicola Stananought, Marie Greene, Jane Sutherland, Corinna Midgley, Evelyn Asiam Saint Francis Hospice, Essex, England

10.1136/bmjspcare-2011-000105.101

Introduction Patients with MND are usually referred to palliative care services late in their disease progression. Reasons for this appear to centre on patients being so distressed at the prospect of palliative care services involvement, that they delay referral for as long as possible. Having identified this as a problem, the hospice embarked on a joint venture with the Motor Neurone Disease Association. The premise of such joint working was to develop an outreach project for local patients with early stage MND. A grant was successfully secured from the $\mathrm{DH}$ (project 64 grant).

Aims The aims of the project were to introduce this cohort of patients/carers to palliative care services at a much earlier stage in their illness and to decrease levels of distress/anxiety associated with a referral. It was also to give the opportunity for patients/carers to meet others in a similar situation and to provide them with information about common problems.

Methods Two programmes were devised, each of 10 sessions, with a different theme per session. Each programme was devised to support 10 new patients/carers, with sessions taking place in the day hospice (hence the title 'a foot in the door').

Results During the first and final sessions of each programme, patients/carers were asked to anonymously score a series of statements concerning their confidence in (and experience in accessing) care, their concerns for the future and feelings about hospice services. Results show that a positive change in all domains occurred.

Conclusion This outreach project had a positive impact on MND patients/carers. The project format can be replicated by other palliative care providers.

A DVD further detailing the project is being made in order to help other palliative care providers develop similar work. 